\title{
Integral part problems derived from a solution of an infimum problem
}

\author{
Pawee Pasteczka and ÁrpÁD SzÁz
}

Abstract. In this paper, we solve the following two integral part problems:

Find all $r \in \mathbb{R}$ satisfying

$$
r^{2}=[r]([r]+1), \quad \text { resp. } \quad r^{2} \leq[r]([r]+1) .
$$

These problems have been mainly motivated by a solution of an infimum problem of Z. Boros and Á. Száz.

Key words and phrases: Real and integral numbers, integral part equations and inequalities.

ZDM Subject Classification: Primary 39B22, 39B62; Secondary 97I10, $97 \mathrm{I} 70$.

\section{Introduction}

In the sequel, we shall use the standard notations $\mathbb{R}, \mathbb{N}, \mathbb{Z}$ and $\mathbb{Q}$ for the sets of all real, natural, integral and rational numbers, respectively.

Moreover, for any $r \in \mathbb{R}$, we shall consider the integral part

$$
[r]=\max \{k \in \mathbb{Z}: \quad k \leq r\}
$$

which exists by an important consequence of a usual axiom system for $\mathbb{R}[1]$.

The work of the second author has been supported by the Hungarian Scientific Research Fund (OTKA) Grant K-111651. 
In $[5,6], Z$. Boros and the second author presented two solutions for the following infimum problem derived from the proofs of some generalized Schwarz inequalities $[3,4]$.

Problem 1.1. For all $a, b \in \mathbb{R}$, determine

$$
F(a, b)=\inf _{n \in \mathbb{N}}\left(a n+\frac{b}{n}\right) .
$$

In one of the solutions, they have proved that

$$
F(a, b)=\left\{\begin{array}{rll}
-\infty & \text { if } \quad a<0, \\
0 & \text { if } \quad a=0 \leq b, \\
a+b & \text { if } \quad b \leq 0 \leq a \quad \text { or } \quad 0<b \leq a
\end{array}\right.
$$

Moreover, if $0<a<b$, then under the notation $r=\sqrt{b / a}$ we have

$$
F(a, b)=a[r]+b /[r] \quad \text { if } \quad r^{2} \leq[r]([r]+1)
$$

and

$$
F(a, b)=a([r]+1)+b /([r]+1) \quad \text { if } \quad[r]([r]+1)<r^{2} .
$$

This solution has motivated us to consider the following two integral part problems.

Problem 1.2. Find all $r \in \mathbb{R}$ satisfying the equality

$$
r^{2}=[r]([r]+1) .
$$

Problem 1.3. Find all $r \in \mathbb{R}$ satisfying the inequality

$$
r^{2} \leq[r]([r]+1)
$$

The latter two problems can also be well motivated by the fact that the integral part function, under the name floor function, is widely used not only in pure mathematics, but also in applied mathematics. ( See, for instance, [7] for some recent notations and applications.) 


\section{A solution for Problem 1.2}

Solution 2.1. Suppose that $r \in \mathbb{R}$, and define

$$
k=[r] .
$$

Then, by the definition of $[r]$, it is clear that

$$
k \in \mathbb{Z} \quad \text { and } \quad k \leq r<k+1,
$$

and thus $0 \leq r-k<1$. Hence, by defining

$$
d=r-k,
$$

we can see that

$$
0 \leq d<1 \quad \text { and } \quad r=k+d .
$$

Moreover, if $r$ is a solution of equation (1), then we can see that $r^{2}=$ $k(k+1)$, and thus

$$
|r|=\sqrt{k(k+1)} .
$$

Hence, if either $k=-1$ or $k=0$, we can see that $|r|=0$, and thus $r=0$. Therefore, $r$ may be zero, but cannot belong to $[-1,0[$. Thus, in the sequel, we have to consider only the cases when either $k \leq-2$ or $1 \leq k$. Namely, $k \in \mathbb{Z}$.

If $1 \leq k$, then $2 \leq k+1$ and $1 \leq k+d=r$, since $0 \leq d$. Therefore,

$$
r=|r|=\sqrt{k(k+1)}=\sqrt{k} \sqrt{k+1}
$$

and

$$
d=r-k=\sqrt{k} \sqrt{k+1}-k=\sqrt{k}(\sqrt{k+1}-\sqrt{k}) .
$$

Hence, by using the identity $a-b=\left(a^{2}-b^{2}\right) /(a+b)$, we can infer that

$$
d=\frac{\sqrt{k}}{\sqrt{k}+\sqrt{k+1}} .
$$

Now, by using the increasingness and a limit property of the function $\varphi$, defined by

$$
\varphi(x)=\frac{\sqrt{x}}{\sqrt{x}+\sqrt{x+1}}=\frac{1}{1+\sqrt{1+1 / x}}
$$


for all $x \in[1,+\infty[$, we can see that

$$
\sqrt{2}-1 \leq d<1 / 2
$$

Thus, in particular $r \notin \mathbb{Z}$. Moreover, we may think that $d$ can get arbitrarily close to $1 / 2$.

While, if $k \leq-2$, then $2 \leq-k, 1 \leq-k-1$ and $r=k+d<-1$, since $d<1$. Therefore,

$$
r=-|r|=-\sqrt{k(k+1)}=-\sqrt{-k(-k-1)}=-\sqrt{-k} \sqrt{-k-1}
$$

and

$$
d=r-k=-\sqrt{-k} \sqrt{-k-1}-k=-\sqrt{-k}(\sqrt{-k-1}-\sqrt{-k}) .
$$

Hence, by using the identity $a-b=\left(a^{2}-b^{2}\right) /(a+b)$, we can infer that

$$
d=\frac{\sqrt{-k}}{\sqrt{-k}+\sqrt{-k-1}} .
$$

Now, by using a limit property and the increasingness of the function $\psi$, defined by

$$
\psi(x)=\frac{\sqrt{-x}}{\sqrt{-x}+\sqrt{-x-1}}=\frac{1}{1+\sqrt{1+1 / x}}
$$

for all $x \in]-\infty,-2]$, we can see that

$$
1 / 2<d \leq 2-\sqrt{2}
$$

Thus, in particular $r \notin \mathbb{Z}$. Moreover, we may think that $d$ can get arbitrarily close to $1 / 2$.

Thus, we have established only some necessary conditions for $k$ and $d$, depending on $k$, so that $r$ could be a solution of equation (1). Actually, by these conditions, we can only state that if $r$ is a solution of (1), then $r$ is in the set

$$
\{0\} \cup\{\sqrt{k(k+1)}\}_{k=1}^{+\infty} \cup\{-\sqrt{k(k+1)}\}_{k=-2}^{-\infty} .
$$

Therefore, in the sequel, we have to determine that which elements of the set $(3)$ are, in fact, solutions of equality (1).

For this, we first note that if

$$
r=\sqrt{k(k+1)}
$$


for some $k \in \mathbb{N}$, then

$$
k=\sqrt{k^{2}}<\sqrt{k(k+1)}=r .
$$

Moreover, by our former similar computation,

$$
r-k=\sqrt{k(k+1)}-k=\frac{\sqrt{k}}{\sqrt{k}+\sqrt{k+1}}<\frac{1}{2}<1 .
$$

Hence, it is clear that

$$
r=k+(r-k)<k+1
$$

also holds. Therefore, we necessarily have $k=[r]$. Now, we can already see that

$$
r^{2}=k(k+1)=[r]([r]+1) .
$$

Therefore, $r$ is a solution of equation (1).

Next, we note that if

$$
r=-\sqrt{k(k+1)}
$$

for some $k \in \mathbb{Z}$ with $k \leq-2$, then

$$
-r=\sqrt{-k(-k-1)}<\sqrt{(-k)^{2}}=-k,
$$

and thus $k<r$. Moreover, by our former similar computation,

$$
r-k=-\sqrt{k(k+1)}-k=\frac{\sqrt{-k}}{\sqrt{-k}+\sqrt{-k-1}} \leq 2-\sqrt{2}<1 \text {. }
$$

Now, we can already see that

$$
r=k+r-k<k+1
$$

also holds. Therefore, we necessarily have $k=[r]$. Now, we can already see that

$$
r^{2}=k(k+1)=[r]([r]+1) .
$$

Therefore, $r$ is a solution of equation (1).

Hence, since $r=0$ is a trivial solution of (1), it is clear that every element of the set (3) is a solution of (1). Thus, the set (3) is precisely the family of all solutions of (1). 
REMARK 2.2. Before the above observations, one can note that if in particular $r \in \mathbb{Z}$, then $[r]=r$. Therefore, if $r$ is, in addition, a solution of (1), then

$$
r^{2}=[r]([r]+1)=r(r+1)=r^{2}+r,
$$

and thus $r=0$. Therefore, the elements of the set $\mathbb{Z} \backslash\{0\}$ cannot be solutions of (1)

REMARK 2.3. Somewhat more strikingly, we can also note that if $r \in \mathbb{Q}$ is a solution of (1), then

$$
r^{2}-[r]([r]+1)=0
$$

holds, with $[r]([r]+1) \in \mathbb{Z}$. Therefore, by a very particular case of $[2$, Theorem 5.3], we necessarily have $r \in \mathbb{Z}$. Thus, by Remark 2.2, we also have $r=0$. Therefore, the elements of the set $\mathbb{Q} \backslash\{0\}$ cannot also be solutions of $(1)$

REMARK 2.4. Moreover, by using the definition of the integral part, one can easily prove that if $r \in \mathbb{R} \backslash \mathbb{Z}$, then

$$
[-r]=-[r]-1
$$

Therefore, if $r$ is, in addition, a solution of (1), then in particular we also have

$$
[-r]([-r]+1)=(-[r]-1)(-[r])=[r]([r]+1)=r^{2}=(-r)^{2} .
$$

Therefore, in this case, $-r$ is also a solution of (1). However, this observation cannot also be used to shorten our former solution.

\section{A solution for Problem 1.3}

Solution 3.1. If $r$ is a solution of inequality (2), then by using the notations of Solution 2.1, we can see that $r^{2} \leq k(k+1)$, and thus

$$
|r| \leq \sqrt{k(k+1)}
$$

Hence, if either $k=-1$ or $k=0$, we can see that $|r|=0$, and thus $r=0$. Therefore, $r$ may be zero, but cannot belong to $[-1,0[$. Thus, in the 
sequel, we shall need only consider the cases when either $k \leq-2$ or $1 \leq k$. Namely, $k \in \mathbb{Z}$.

If $1 \leq k$, then $2 \leq k+1$ and $1 \leq k+d=r$, since $0 \leq d$. Therefore,

$$
r=|r| \leq \sqrt{k(k+1)}=\sqrt{k} \sqrt{k+1}
$$

and

$$
d=r-k \leq \sqrt{k} \sqrt{k+1}-k=\sqrt{k}(\sqrt{k+1}-\sqrt{k}) .
$$

Hence, as in Solution 2.1, we can infer that

$$
d \leq \frac{\sqrt{k}}{\sqrt{k}+\sqrt{k+1}}<\frac{1}{2} .
$$

Moreover, we may think that $d$ can get arbitrarily close to $1 / 2$.

While, if $k \leq-2$, then $2 \leq-k, 1 \leq-k-1$ and $r=k+d<-1$, since $d<1$. Therefore,

$$
r=-|r| \geq-\sqrt{k(k+1)}=-\sqrt{-k(-k-1)}=-\sqrt{-k} \sqrt{-k-1}
$$

and

$$
d=r-k \geq-\sqrt{-k} \sqrt{-k-1}-k=-\sqrt{-k}(\sqrt{-k-1}-\sqrt{-k}) .
$$

Hence, as in Solution 2.1, we can infer that

$$
d \geq \frac{\sqrt{-k}}{\sqrt{-k}+\sqrt{-k-1}}>\frac{1}{2} .
$$

Moreover, we may think that $d$ can get arbitrarily close to $1 / 2$.

Thus, we have established only some necessary conditions for $k$ and $d$, depending on $k$, so that $r$ could be a solution of inequality (2). Actually, by these conditions, we can only state that if $r$ is a solution of (2), then $r$ is in the set

$$
\{0\} \cup \bigcup_{k=1}^{\infty}[k, \sqrt{k(k+1)}] \cup \bigcup_{k=-2}^{-\infty}[-\sqrt{k(k+1)}, k+1[.
$$

Therefore, in the sequel, we have to determine that which elements of the set (4) are, in fact, solutions of inequality (2). 
For this, we first note that if

$$
r \in[k, \sqrt{k(k+1)}]
$$

for some $k \in \mathbb{N}$, then

$$
k \leq r \leq \sqrt{k(k+1)} .
$$

Thus, by our former similar computation,

$$
r-k \leq \sqrt{k(k+1)}-k=\frac{\sqrt{k}}{\sqrt{k}+\sqrt{k+1}}<\frac{1}{2} .
$$

Hence, we can already infer that

$$
k \leq r=k+(r-k)<k+1 / 2<k+1,
$$

and thus $k=[r]$. Now, since

$$
1 \leq k \leq r \leq \sqrt{k(k+1)},
$$

we can also see that

$$
r^{2} \leq k(k+1)=k^{2}+k=[r]^{2}+[r],
$$

and thus (2) also holds. Therefore, $r$ is a solution of (2).

Next, we note that if

$$
r \in[-\sqrt{k(k+1)}, k+1[
$$

for some $k \in \mathbb{Z}$ with $k \leq-2$, then

$$
-\sqrt{k(k+1)} \leq r<k+1,
$$

Thus, by our former similar computation,

$$
\frac{1}{2}<\frac{\sqrt{-k}}{\sqrt{-k}+\sqrt{-k-1}}=-\sqrt{k(k+1)}-k \leq r-k .
$$

Hence, we can already infer that

$$
k<k+1 / 2<k+r-k=r<k+1,
$$

and thus $k=[r]$. Now, since

$$
1 \leq-k-1 \leq-r \leq \sqrt{k(k+1)},
$$


we can also see that

$$
r^{2} \leq k(k+1)=k^{2}+k=[r]^{2}+[r],
$$

and thus (2) also holds. Therefore, $r$ is a solution of (2).

Hence, since $r=0$ trivially satisfies (2), it is clear that every element of the set (4) is a solution of (2). Thus, the set (4) is precisely the family of all solutions of $(2)$.

Now, analogously to Remarks 2.2 and 2.4, we can also easily establish the following assertions.

REMARK 3.2. If in particular $r \in \mathbb{Z}$ such that $r$ is a solution of (2), then

$$
r^{2} \leq[r]([r]+1)=r(r+1)=r^{2}+r,
$$

and thus $0 \leq r$. Therefore, each non-negative integer is a solution of (2), but the negative integers cannot be solutions of (2).

REMARK 3.3. If in particular if $r \in \mathbb{R} \backslash \mathbb{Z}$ such that $r$ is a solution of (2), then

$$
[-r]([-r]+1)=(-[r]-1)(-[r])=[r]([r]+1) \geq r^{2}=(-r)^{2} .
$$

Therefore, in this case, $-r$ is also a solution of (2). However, this observation cannot also be used to shorten our former solution.

\section{Suggestions for some further investigations}

Inequality (2) can be reformulated in the more instructive forms that

$$
r^{2}-[r]^{2} \leq[r] \quad \text { and } \quad r^{2}+(1 / 2)^{2} \leq([r]+1 / 2)^{2} .
$$

Therefore, instead of Problems 1.2 and 1.3, one may also naturally consider the following two more general problems.

Problem 4.1. Find all $r, s \in \mathbb{R}$ satisfying the equality

$$
r^{2}+s^{2}=([r]+s)^{2} .
$$


Problem 4.2. Find all $r, s \in \mathbb{R}$ satisfying the inequality

$$
r^{2}+s^{2} \leq([r]+s)^{2} .
$$

However, the solutions of the latter two problems would require some more difficult computations which are certainly not suitable for class room presentation.

Therefore, it seems to be more interesting to look for solutions $r$ of

$$
r^{2}=\varphi(r)(\varphi(r)+1), \quad \quad \text { resp. } \quad r^{2} \leq \varphi(r)(\varphi(r)+1),
$$

where $\varphi$ is a fixed, reasonable function of $\mathbb{R}$ to itself.

For instance, for any $r \in \mathbb{R}$, we may naturally take

$$
\varphi(r)=\langle r\rangle=r-[r] .
$$

Thus, $\varphi(r)$ is just the fractional part of $r$.

Moreover, for any $r \in \mathbb{R}$, we may also naturally take

$$
\varphi(r)=[r] \quad \text { if } \quad\langle r\rangle \leq 1-\langle r\rangle \quad \text { and } \quad \varphi(r)=[r]+1 \quad \text { if } \quad 1-\langle r\rangle\langle\langle r\rangle .
$$

Thus, $\varphi(r)$ is just the nearest integer to $r$. That is, $d(r, \mathbb{Z})=d(r, \varphi(r))$.

Historical notes. By the Wikipedia, the integral part of a real number $x$ was first considered by A.-M. Legendre in 1798. While, the notation $[x]$ was first introduced by C.F. Gauss in 1808. Later, a great number of interesting formulas has been established for the integral part.

For instance, S. Ramanujan, among other similar formulas for the integral part, noticed that

$$
[1 / 2+\sqrt{n+1 / 2}]=\left[1 / 2+\sqrt{n+(1 / 2)^{2}}\right]
$$

for all $n \in \mathbb{N}$. (Thus, for instance, the solutions $r$ of the equation

$$
[s+\sqrt{r+s}]=\left[s+\sqrt{r+s^{2}}\right],
$$

with $s=1 / 2$, can also be hopefully looked for.)

Moreover, to illustrate the advantage of Fourier series over the Taylor and Laurent ones at undergraduate courses, it is customary to prove that

$$
[x]=x-\frac{1}{2}+\frac{1}{\pi} \sum_{k=1}^{\infty} \frac{\sin (2 \pi k x)}{k}
$$


for all $x \in \mathbb{R}$. (Note that in the above two important equalities, as well as an instructive reformulation of our Problem 1.2, the number 1/2 appears.)

In a 1962 book, K. E. Iverson introduced the modern name "floor function" and notation $\lfloor x\rfloor$ instead of "integral part function" and $[x]$. However, in the present paper, for the sake of the understanding of a wider audience, it seemed more convenient to use the old-fashioned terminology and notation.

Acknowledgement. The authors are indebted to Gy. Maksa for suggesting the use of fractional part instead of the integral one, and B. Sury for sending us a sketch of a somewhat different, independent solution for Problem 1.3.

\section{References}

[1] Z. Boros and Á. Száz, The smallest denominator function and the Riemann function, Acta Math. Acad. Paedagog. Nyházi. (N.S.) 14(1998), 1-17.

[2] Z. Boros and Á. Száz, Some number theoretic applications of the smallest denominator function, Acta Math. Acad. Paedagog. Nyházi. (N.S.) 15 (1999), 19-26.

[3] Z. Boros and Á. Száz, A weak Schwarz inequality for semi-inner products on groupoids, Rostock. Math. Kolloq. 71 (2016), 28-40.

[4] Z. Boros and Á. Száz, Generalized Schwarz inequalities for generalized semi-inner products on groupoids can be derived from an equality, Novi Sad J. Math. 47 (2017), $177-188$.

[5] Z. Boros and Á. Száz, Solutions to Problem-1, Math. Student 86, No. 3-4 (2017), 130-132.

[6] Z. Boros and Á. Száz, Infimum problems derived from the proofs of some generalized Schwarz inequalities, manuscript.

[7] J. C. Lagarias, T. Murayama and D. H. Richman, Dilated floor functions that commute, Amer. Math. Montly 123 (2016), 1033-1038.

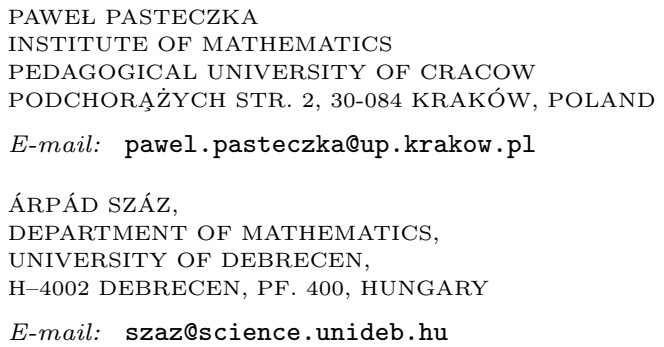

